\title{
MEASUREMENT OF THE UNSTEADY LIFT OF THICK AIRFOILS IN INCOMPRESSIBLE TURBULENT FLOW
}

\author{
Peter D. Lysak*, Dean E. Capone, and Michael L. Jonson \\ Applied Research Laboratory \\ The Pennsylvania State University \\ P.O. Box 30 \\ State College, PA 16804
}

\begin{abstract}
The unsteady lift spectrum for airfoils in turbulent flow has been measured in a water tunnel experiment. The results provide validation data for analytical models that account for the effect of airfoil thickness on the high frequency gust response. A series of four airfoils with elliptical leading edge profiles and thickness-to-chord ratios ranging from 8 to 16 percent were tested in grid-generated turbulence. The turbulent velocity spectrum was measured using Laser Doppler Velocimetry, and was found to be reasonably well approximated by an isotropic, homogeneous turbulence model. The unsteady force measurement setup and calibration procedure were designed to minimize the effect of system resonances, and contamination from facility vibration was reduced using a multiple coherence noise removal technique. Measurements of the unsteady lift spectrum were made at six speeds over the Reynolds number range 0.5 to 1.6 million, and the results were collapsed into a nondimensional force spectrum for the non-dimensional frequency range $1<f c / U_{\infty}<10$. The experimental results agree very well with theory and confirm the importance of accounting for thickness to avoid overprediction of the forces in the high frequency range.
\end{abstract}

Keywords: turbulence ingestion noise; airfoil gust response; unsteady force measurement

* Corresponding author. E-mail address: pdl103@psu.edu 


\section{List of Tables}

$1 \quad$ LDV measured mean and rms velocity profiles. . . . . . . . . . . . . . 12

2 Integral length scales determined from LDV correlation measurements. . . . 15

\section{List of Figures}

1 Schematic of the experimental setup. . . . . . . . . . . . . 8

2 Schematic of the water tunnel test section hardware. . . . . . . . . . . . 10

3 Measured longitudinal correlation function based on the streamwise velocity. 14

4 Measured transverse correlation function based on the spanwise velocity. . . 15

5 Measured longitudinal velocity spectrum based on the streamwise velocity. . 17

6 Measured transverse velocity spectrum based on the spanwise velocity. . . . 18

$7 \quad$ Calibration of the summed force gage response. . . . . . . . . . . . . . 20

8 Example accelerometer spectra. . . . . . . . . . . . . . . . . 22

9 Example results of the coherent noise removal technique. . . . . . . . . . . . 22

10 Example signal-to-noise results. . . . . . . . . . . . . . . . . . . . 24

11 Collapse of the measured force spectra to a single non-dimensional curve. . 25

12 Background noise correction applied to the $12.5 \%$ thick airfoil. . . . . . . 26

13 Background noise correction applied to the $16 \%$ thick airfoil. . . . . . . . 26

14 Force spectrum results for the $8 \%$ thick airfoil. . . . . . . . . . . . . 27

15 Force spectrum results for the $10 \%$ thick airfoil. . . . . . . . . . . . . 28

16 Force spectrum results for the $12.5 \%$ thick airfoil. . . . . . . . . . . . 28

17 Force spectrum results for the $16 \%$ thick airfoil. . . . . . . . . . . . . . . 29 


\section{Nomenclature}

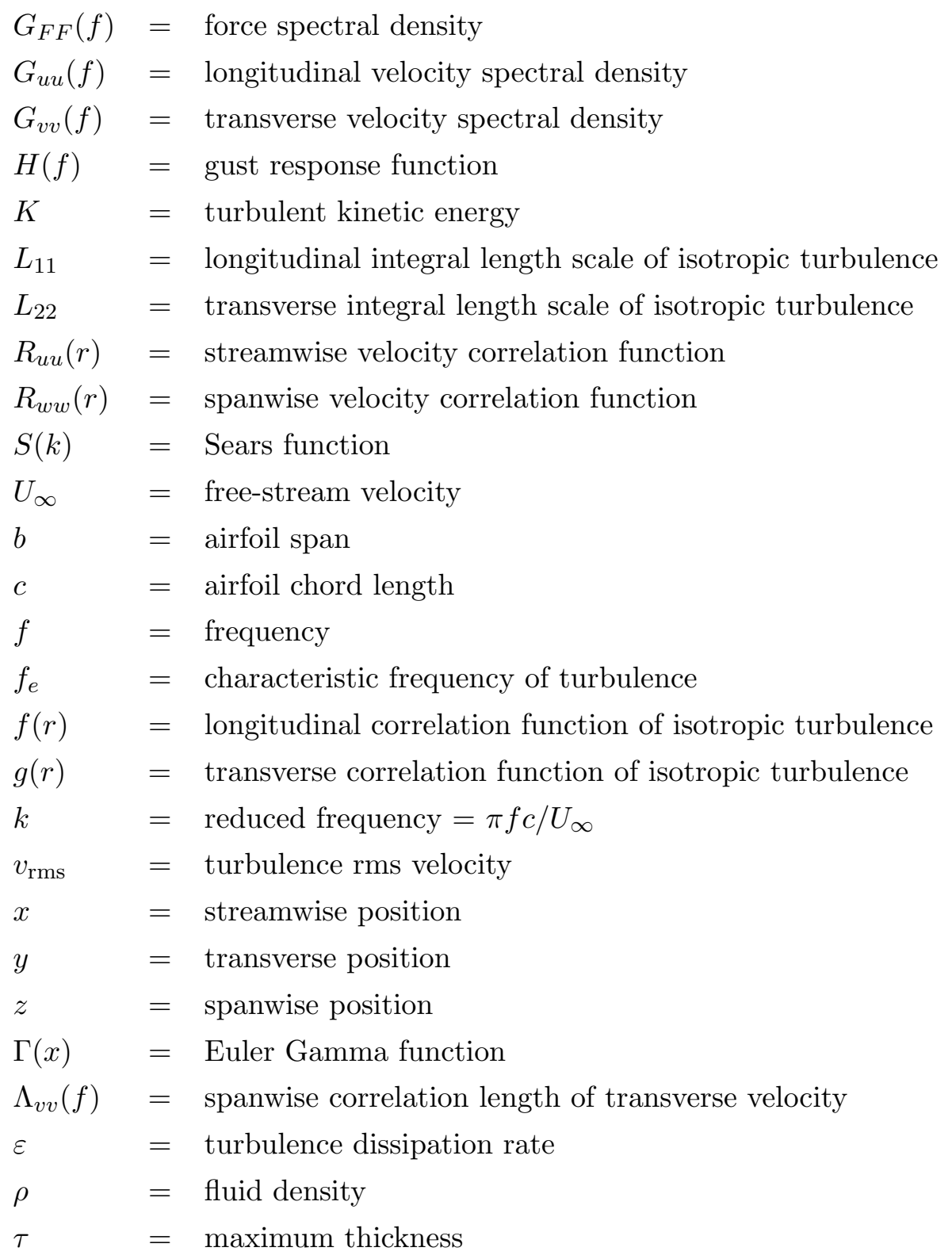




\section{Introduction}

The unsteady lift forces that act on an airfoil in turbulent flow are influenced by the airfoil thickness at high frequencies. One of the earliest demonstrations of this effect was by Paterson and Amiet (1977), who measured the noise generated by a NACA 0012 airfoil and compared the results to the turbulence ingestion noise theory of Amiet (1975). They found the theory to be accurate except at low speeds and high frequencies, where the theory overpredicted the noise. This was due to the limitations of the flat plate gust response model used in the theory, which does not account for the distortion of the incident gusts by the curvature of the streamlines near the airfoil leading edge. This effect becomes important when the gust wavelengths (which equal $U_{\infty} / f$ ) are smaller than the thickness of the airfoil.

Further evidence of the thickness effect was provided by Olsen and Wagner (1982), who made noise measurements using airfoils with thickness-to-chord ratios of 3,13 , and 37 percent. Their data only provided a relative comparison of the noise, as an inhomogeneous turbulent jet was used for the inflow and measurements of the turbulence spectrum were not made. However, by testing such a wide thickness range, they were able to clearly show that the noise was reduced with increasing thickness. Also, the amount of reduction was observed to increase with increasing frequency.

More recent noise measurements have confirmed these trends. Oerlemans and Migliore (2004) tested a series of wind turbine airfoils and found that the incident turbulence noise decreased with increasing leading edge radius. Moreau et al. (2005) studied a $3 \%$ thick flat plate, a 4\% thick cambered airfoil, and a NACA 0012 airfoil. They found that the noise from the flat plate and the thin cambered airfoil were well predicted by Amiet's theory, with only slight differences between the two cases. However, a more significant difference occurred for the NACA 0012 airfoil, which had up to $10 \mathrm{~dB}$ lower noise at high frequencies. Further analysis of these results and comparisons with other data are given in a review by Roger and Moreau (2010). Finally, Devenport et al. (2010) tested several airfoils of different chord lengths and thickness profiles. They also found that the measured noise was significantly lower than the flat plate model predictions, and that a model which accounted for thickness effects fit the data much better. In addition, all of these researchers investigated the effect of angle of attack on the noise. While measurable differences were observed, it was generally found that angle of attack effects were much less important than thickness.

One property that these recent measurements have in common is that they were all made at relatively low Mach numbers ( $M \sim 0.1$, based on free-stream velocity). This is significant because the high frequency spectrum depends not only on the non-dimensional 
frequency $f c / U_{\infty}$, but also on the ratio of the acoustic wavelength to the airfoil chord, which equals $\left(M f c / U_{\infty}\right)^{-1}$. For a given non-dimensional frequency, the acoustic wavelength-tochord ratio decreases with increasing Mach number. This changes the unsteady lift because the influence of the gust interaction is limited to a few acoustic wavelengths downstream of the leading edge. It also changes the acoustic radiation due to phase interference effects from the distributed dipole sources. The net result is that the slope of the gust response spectrum becomes steeper as the Mach number increases (Amiet, 1976). This means that the turbulence ingestion noise will be overtaken by other high frequency noise sources such as trailing edge noise at lower frequencies as the Mach number increases, possibly making the range affected by thickness less significant. Therefore, the thickness effect tends to be more important at low Mach numbers.

In this paper, experimental results from a water tunnel test are presented. Compared to earlier measurements involving the radiated noise, the focus of these measurements is the source of the noise, which is the unsteady lift spectrum. This helps to clarify the incompressible flow mechanisms that are responsible for the gust distortion and unsteady lift, without additional complications from acoustic non-compactness and radiation effects. In addition, the results are relevant for problems involving flow-induced vibration.

The experiment consisted of a series of unsteady force spectrum measurements for airfoils with thickness-to-chord ratios ranging from 8 to 16 percent, operating in grid-generated turbulence. The airfoils were based on the NACA 65 series, which has an elliptical leading edge thickness profile up to about $40 \%$ chord. This shape is widely used because it has low drag for small angles of attack, but has not been examined in previous turbulence ingestion studies. The objective of the experiment was to develop and validate a modified gust response model that accounts for thickness for this family of airfoils.

The measured unsteady forces depend on both the characteristics of the inflow turbulence and the airfoil gust response. A brief review of the theory used to combine these factors is given in Sec. 2. Then, after describing the setup of the experiment in Sec. 3, the Laser Doppler Velocimetry (LDV) measurements used to characterize the inflow will be discussed in Sec. 4. The measurement techniques used to obtain the airfoil force spectrum are described in detail in Sec. 5, followed by examination of the thickness effect in Sec. 6 .

\section{Unsteady Lift from Inflow Turbulence}

The theory used to relate the fluctuating forces to the inflow turbulence is based on the model developed by Amiet (1975). In the current work, it is expressed in a form representing the unsteady lift in incompressible flow rather than the radiated noise in compressible flow. 
The model involves three factors: 1) the spectrum of the turbulent velocity component normal to the airfoil (i.e., the gust), 2) the spanwise coherence of the turbulence, and 3) the transfer function that relates the gust to the airfoil unsteady lift. It is assumed that the gusts are small perturbations compared to the free-stream velocity, and that the overall response can be found by superposition of the response from individual sinusoidal components. In addition, the gust response is based on two-dimensional unsteady airfoil theory, and neglects any three-dimensional effects that might occur at the ends of the span.

The spectrum of the total force acting on the airfoil can be expressed in the form

$$
G_{F F}(f)=G_{v v}(f) \Lambda_{v v}(f)|H(f)|^{2} b
$$

where $G_{v v}(f)$ is the spectral density of the turbulent velocity upstream of the airfoil, $\Lambda_{v v}(f)$ is the spanwise correlation length of the turbulent velocity, $H(f)$ is the gust response function, and $b$ is the airfoil span. Assuming isotropic, homogeneous turbulence, the von Kármán model (Hinze, 1975) provides formulas for the turbulence functions. For components perpendicular to the free-stream, the turbulent velocity spectrum is given by

$$
G_{v v}(f)=2 \frac{v_{\mathrm{rms}}^{2} L_{11}}{U_{\infty}}\left[\frac{f_{e}^{2}+\frac{8}{3} f^{2}}{f_{e}^{2}+f^{2}}\right]\left[1+\left(\frac{f}{f_{e}}\right)^{2}\right]^{-5 / 6}
$$

where $v_{\text {rms }}$ is the root mean square velocity, $L_{11}$ is the longitudinal integral length scale of the turbulence, and $f_{e}$ is the characteristic frequency of the energy-containing eddies, which is defined as

$$
f_{e}=\frac{1}{2 \sqrt{\pi}} \frac{\Gamma\left(\frac{5}{6}\right)}{\Gamma\left(\frac{1}{3}\right)} \frac{U_{\infty}}{L_{11}} \approx 0.12 \frac{U_{\infty}}{L_{11}}
$$

Using the same model, the frequency-dependent spanwise correlation length of the turbulence is

$$
\Lambda_{v v}(f)=\frac{16 L_{11}}{9}\left[\frac{\Gamma\left(\frac{1}{3}\right)}{\Gamma\left(\frac{5}{6}\right)}\right]^{2}\left[\frac{f^{2}}{f_{e}^{2}+\frac{8}{3} f^{2}}\right]\left[1+\left(\frac{f}{f_{e}}\right)^{2}\right]^{-1 / 2}
$$

Finally, the gust response function for a flat plate airfoil is found from the two-dimensional unsteady airfoil theory of Sears (1941):

$$
|H(f)|^{2}=\left(\pi \rho c U_{\infty}\right)^{2}\left|S\left(\pi f c / U_{\infty}\right)\right|^{2}
$$

where $S(k)$ is the Sears function. While an exact evaluation of the Sears function involves Hankel functions, an excellent approximation to $|S(k)|^{2}$ that is accurate to within nearly $0.1 \mathrm{~dB}$ is given by

$$
|S(k)|^{2} \approx\left[\frac{1}{1+(2 \pi k)^{m}}\right]^{1 / m}
$$


with $m=1.3$. When the effect of airfoil thickness is included, the gust response $|H(f)|^{2}$ needs to be modified. Based on calculations of the gust distortion by the mean flow around the airfoil, and using an unsteady lift calculation methodology designed to capture the high frequency asymptote of the leading edge interaction, Lysak et al. (2013) proposed a modified gust response function that includes a quasi-steady correction factor and highfrequency attenuation function:

$$
|H(f)|^{2}=\left(\pi \rho c U_{\infty}\right)^{2}\left(1+0.8 \frac{\tau}{c}\right)^{2}\left|S\left(\pi f c / U_{\infty}\right)\right|^{2} \exp \left[-\beta(\tau) \frac{f c}{U_{\infty}}\right]
$$

where

$$
\beta(\tau) \approx 0.75(\tau / c)+12(\tau / c)^{2}
$$

Similar exponential attenuation modifications were developed by Gershfeld (2004) and Moriarty et al. (2005), but the thickness factor $\beta(\tau)$ shown above is specifically for airfoils with an elliptical leading edge thickness profile like the NACA 65 series. Different values of $\beta$ would be appropriate for other leading edge shapes.

In designing an experiment that approximates the idealized configuration assumed in the Amiet theory, a key observation is that Eq. (1) represents the summation of multiple independent sources of unsteady force, provided that the spanwise correlation length is small relative to the span. This condition can be met by generating inflow turbulence with an integral length scale much smaller than the span. The assumption also becomes increasingly valid as the frequency increases, because the spanwise correlation length gets smaller at higher frequencies. Furthermore, when the total force results from mulitple independent sources, small variations in the turbulence statistics tend to average out in the spanwise summation, so the force can be predicted using spanwise averaged parameters.

\section{$3 \quad$ Experimental Setup}

The experiment was conducted in the 12-inch diameter water tunnel at the Applied Research Laboratory of the Pennsylvania State University (Lehman, 1959). The facility is a closed circuit, closed jet water tunnel which can be configured with either a $305 \mathrm{~mm}$ (12 inch) diameter circular test section or a $114 \mathrm{~mm} \times 508 \mathrm{~mm}(4.5 \times 20$ inch $)$ rectangular test section. For this experiment, the rectangular test section was used to get a two-dimensional environment in which to test the airfoils. Testing in a water tunnel helped ensure that the acoustic wavelengths were long compared to the airfoil chord for the entire frequency range of interest, so the results could be compared to incompressible flow theory.

The test setup is shown in Fig. 1. The span of the airfoils was equal to the distance between the top and bottom walls $(114 \mathrm{~mm})$, and the chord length of the airfoils was 160 


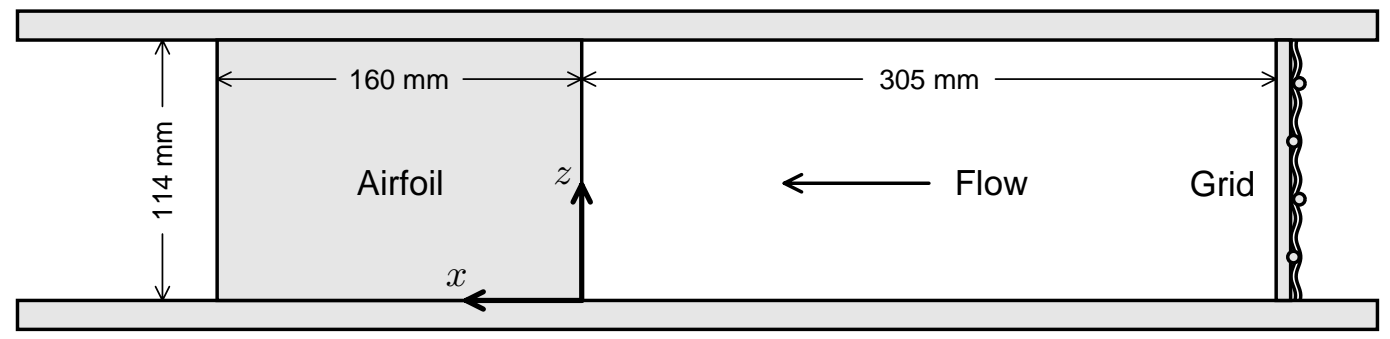

Figure 1: Schematic of the experimental setup. Note the LDV measurements used to characterize the inflow turbulence spectrum were made at the location of the airfoil leading edge, without the airfoil installed.

mm. The selection of the chord length was a compromise between desiring a long chord length in order to maximize the amplitude of the unsteady forces and improve the signalto-noise ratio of the measurements, and wanting a short chord length to increase the aspect ratio of the airfoil and reduce the impact of three-dimensional flow near the wall junctions. Because the focus of the experiment was high frequency unsteady forces due to small scale turbulence, the spanwise correlation lengths involved are small, making it possible to use a relatively short aspect ratio (about 0.7 ) and still have a two-dimensional gust response over most the span. The airfoils were mounted approximately in the center of the test section, giving a distance of about 1.5 chord lengths to the side walls, so there was minimal blockage effect. A series of four airfoils based the NACA 65 $1 \mathrm{~A}^{-012}$ profile (Abbott and von Doenhoff, 1959) were tested. The airfoil profile was scaled to vary the thickness-to-chord ratio to 8 , 10, 12.5, and 16 percent. The airfoils were made out of aluminum using wire electrical discharge machining (EDM) with a specified tolerance of $0.1 \mathrm{~mm}$ and polished to a smooth finish. No boundary layer tripping devices were used, because the inflow turbulence was sufficient to force transition of the boundary layer.

A turbulence-generating grid was mounted at the beginning of the test section, which was $305 \mathrm{~mm}$ (12 inches) upstream of the airfoil leading edge. The grid consisted of a wire mesh with a $25.4 \mathrm{~mm}$ (1 inch) grid spacing made from $3 \mathrm{~mm}$ diameter rods. The rods had a wavy pattern with an amplitude around $1 \mathrm{~mm}$ and wavelength about $17 \mathrm{~mm}$, which helped to induce three-dimensional turbulent mixing of the wakes. One of the most important criteria in selecting the grid was that the peak of the turbulent velocity spectrum be located in the high frequency region $\left(f c / U_{\infty} \gg 1\right)$ where the effect of airfoil thickness is important. This was achieved because the integral length scale of the turbulence, being of the same order of magnitude as the diameter of the rods, was much smaller than the airfoil chord. Another characteristic of the grid was that the mesh was welded onto vertical 
support beams which were $3 \mathrm{~mm}$ thick and $10 \mathrm{~mm}$ wide in the flow direction. Because of the support beams, the grid produced slightly higher turbulent fluctuations in the streamwise direction than in the spanwise direction, as revealed by the velocity measurements discussed below.

A schematic showing how the airfoils were installed in the test section is shown in Fig. 2. To measure the unsteady lift, the airfoils were bolted into rectangular bases, which were fastened to the top and bottom aluminum window inserts of the test section through piezoelectric force gages. A total of four force gages (PCB Piezotronics model 201B03) were used, with two on the top (at the 1/4-chord at 3/4-chord positions) and two on the bottom. The setup was designed so the force gages (along with the soft beryllium copper attachment screws) were the only attachment points connecting the airfoil to the water tunnel frame, in order to prevent any shorting paths which would bias the force measurements. As part of the installation, the force gages were preloaded to approximately $1100 \mathrm{~N}$, which was more than two orders of magnitude larger than any of the unsteady loads expected during the test. Once the force gages were preloaded, they remained in place for the duration of the experiment, and did not need to be disturbed to change the airfoils. The top and bottom window inserts had cutouts so the airfoil bases were flush with the test section walls, and the sensor wires were taken out through glands in the top and bottom cover plates, which sealed the test section. Additional details on the experimental setup can be found in Lysak (2011).

\section{Velocity Measurements}

While the main objective of the experiment was to investigate the unsteady forces, in order to predict the force spectrum it is necessary to model the turbulent velocity spectrum, as represented by Eqs. (2) and (4). To verify that the assumed model was representative of the turbulence in the test, and to provide accurate parameters for the model, a Laser Doppler Velocimetry (LDV) system was used to measure the flow downstream of the turbulencegenerating grid. As noted in Sec. 2, the unsteady force spectrum is related to the spanwise average of the turbulence spectra. Therefore, the objective of the velocity measurements was to obtain appropriate spanwise averaged parameters to use in the model.

The LDV equipment was manufactured by Thermo Systems Incorporated (TSI). A dual-beam, two-color probe was used to obtain the streamwise and spanwise velocity components using the back-scatter method. To increase the data rate, small glass spheres (approximately $10 \mu \mathrm{m}$ in diameter) were added to the water. Velocity measurements were made at several points along the leading edge of the airfoil (the airfoil was not installed 


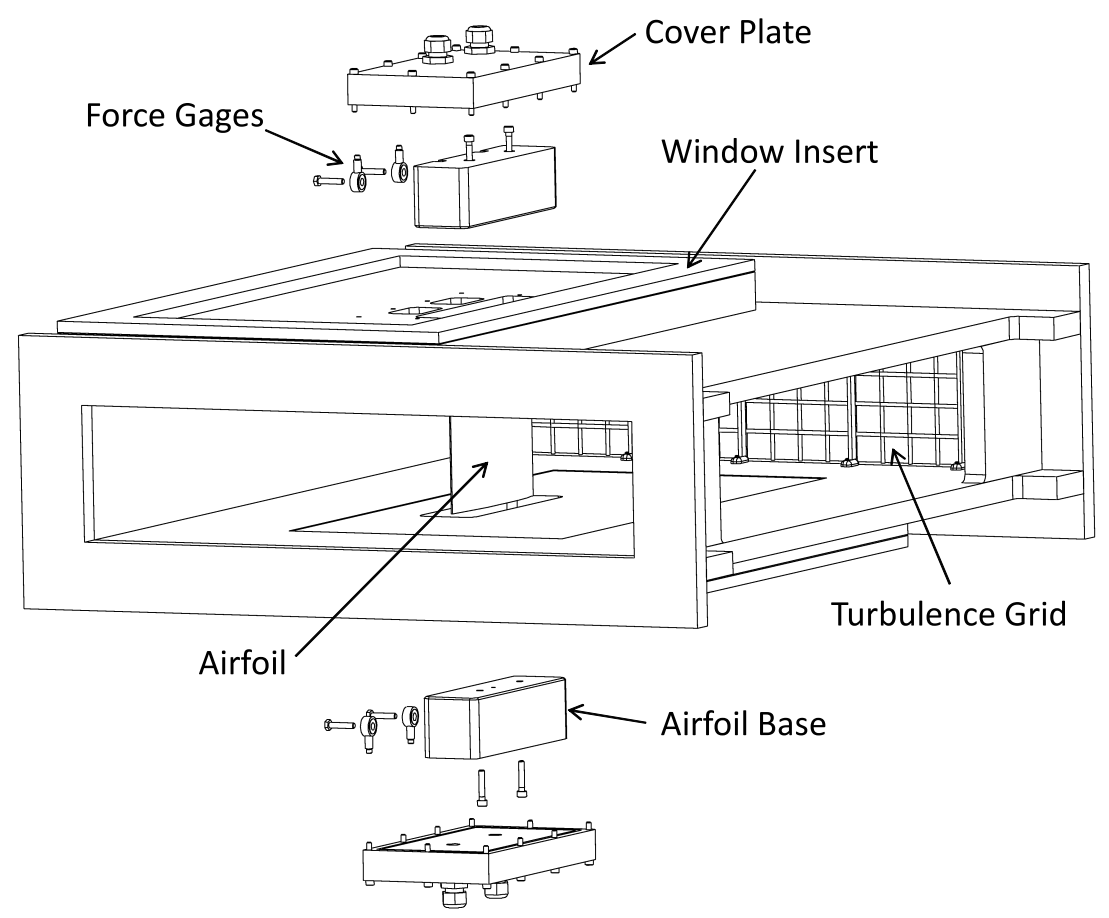

Figure 2: Schematic of the water tunnel test section hardware. Note the drawing of the turbulence grid is only approximate and does not show the waviness of the rods. 
while these measurements were made). Several thousand samples were acquired to get the mean flow statistics, and around 500,000 samples were used for autocorrelation and spectral measurements.

It should be noted that the airfoil forces result mainly from the velocity fluctuations normal to the chord. This component was not measured by the LDV system because there was no optical access for that direction. However, the unsteady force model is also based on the assumption of isotropic turbulence, so the statistics used in the model can be characterized from the longitudinal and transverse correlations and spectra that were obtained from the horizontal (streamwise) and vertical (spanwise) velocity measurements.

\subsection{Speed Calibration}

One of the uses of the LDV measurements was to calibrate the free-stream velocity of the water tunnel. Typically, the free-steam velocity is determined from the pressure difference between a total pressure probe upstream of the water tunnel nozzle and a static pressure port on the test section wall. However, in this experiment, the static pressure port was located downstream of the grid, so it was affected by the pressure drop associated with the resistance of the grid. Because the tunnel control system is based on the pressure difference reading, the nominal free-stream velocities were set according to these indicated values. To determine the correct velocities for post-processing the data, a set of LDV measurements were made over the range of speeds used in the unsteady force tests. A linear least-squares fit to the data indicated that the correct free-stream velocity could be found by multiplying the nominal velocity by 0.84 . As an additional check, a mean velocity survey was also made without the turbulence grid installed. In that case, the measured mean velocity matched the indicated speed from the water tunnel control system to within one percent.

\subsection{Velocity Profiles}

The turbulence-generating grid produced a nearly uniform mean velocity and approximately homogeneous turbulence field. Table 1 lists the measured mean streamwise $(U)$ and vertical $(W)$ velocity components, along with the corresponding rms values ( $u$ and $w$ ). The data in the table represent an average of four spanwise surveys, two taken at a nominal velocity of $3.05 \mathrm{~m} / \mathrm{s}(10 \mathrm{ft} / \mathrm{s})$ and two at $7.62 \mathrm{~m} / \mathrm{s}(25 \mathrm{ft} / \mathrm{s})$. The spanwise variation in the mean streamwise velocity is less than two percent. Some of this variation results from the inherent unsteadiness of the water tunnel drive system and the fact that each point was measured at a different time. No systematic spatial pattern from the grid was observed when comparing repeat surveys, so the effect of the grid geometry on the spatial non-uniformity appears 
Table 1: LDV measured mean and rms velocity profiles.

\begin{tabular}{ccccc}
\hline$z / b$ & $U / U_{\infty}$ & $W / U_{\infty}$ & $u / U_{\infty}$ & $w / U_{\infty}$ \\
\hline 0.2 & 1.010 & -0.026 & 0.0485 & 0.0428 \\
0.3 & 0.993 & -0.016 & 0.0463 & 0.0378 \\
0.4 & 1.014 & -0.011 & 0.0474 & 0.0401 \\
0.5 & 1.001 & -0.011 & 0.0472 & 0.0405 \\
0.6 & 1.009 & -0.012 & 0.0472 & 0.0407 \\
0.7 & 1.013 & -0.008 & 0.0489 & 0.0410 \\
0.8 & 0.989 & 0.006 & 0.0457 & 0.0383 \\
\hline Average & 1.004 & -0.011 & 0.0473 & 0.0402 \\
\hline
\end{tabular}

to be less than the measurement uncertainty. A small mean vertical velocity (about one percent of free-stream) was measured, which is most likely an indication of the alignment error of the system rather than an actual characteristic of the flow. Therefore, it is reasonable to conclude that the mean velocity profile is essentially uniform outside the thin boundary layers near the walls. (Previous testing in the facility found that the boundary layer thickness is on the order of $10 \mathrm{~mm}$, but limited optical access prevented this from being measured in the current setup.)

The rms values are also fairly uniform over the airfoil span, with $\pm 6 \%$ variation. More variation in these quantities is to be expected due to the longer averaging time required for these statistics to converge. A slight departure from isotropic turbulence was observed, with the streamwise rms levels being about $15-20 \%$ higher than the spanwise values. This is most likely due to the vertical support bars which block the streamwise flow but do not generate spanwise disturbances.

The rms values are in reasonably good agreement with the estimated levels for grid turbulence using the empirical formula of Mohamed and LaRue (1990):

$$
\left(\frac{v_{\mathrm{rms}}}{U_{\infty}}\right)^{2}=A\left(\frac{x}{M}\right)^{-1.3}
$$

where $x$ is the distance downstream of the grid, $M$ is the mesh size, and the empirical constant $A$ varies from about 0.035 to 0.055 depending on the drag coefficient of the grid. Using $M=25.4 \mathrm{~mm}$ and $x=305 \mathrm{~mm}$ gives an estimated turbulence intensity $v_{\mathrm{rms}} / U_{\infty}$ between $3.7 \%$ and $4.7 \%$, which brackets the measured results. 


\subsection{Correlation Functions and Integral Length Scales}

The primary use of the LDV system was to measure turbulence correlation functions and spectra. Unlike typical spectrum analyzers which use a fixed sampling rate, the occurrence of LDV samples are Poisson distributed in time. An established method (Benedict, 2000) for computing correlations and spectra is to compute the time lag between all the possible sample pairs, and then sort the velocity products of these pairs into fixed-width bins. The average value in each bin directly gives the temporal autocorrelation, and the autospectrum is then found by taking the Fourier transform of the autocorrelation. Further details of the data processing method are given by Petrie (1995). The optimum value for the bin width depends on the average data rate. To maintain equal normalized frequency resolution at both speeds, a bin width of $0.25 \mathrm{~ms}$ was used in calculating the velocity correlations for the $3.05 \mathrm{~m} / \mathrm{s}(10 \mathrm{ft} / \mathrm{s})$ data, while a bin width of $0.1 \mathrm{~ms}$ was used for the $7.62 \mathrm{~m} / \mathrm{s}(25 \mathrm{ft} / \mathrm{s})$ data. The total number of bins was 500 in both cases.

The measured temporal autocorrelation was used to infer the spatial correlation of the turbulence. Using the frozen convection assumption, which is valid for relatively low turbulence intensities, the effective spatial separation is found by multiplying the time lag by the measured mean streamwise velocity. The longitudinal correlation $f(r)$ and transverse correlation $g(r)$ can be obtained from the streamwise and spanwise velocity components, respectively. The decay rates of the correlation functions are related to the longitudinal and transverse integral length scales, $L_{11}$ and $L_{22}$. For isotropic, homogeneous turbulence, $L_{22}=\frac{1}{2} L_{11}$, so only a single length scale $\left(L_{11}\right)$ is needed to characterize the spatial correlation of the turbulence.

To determine the integral length scale of the grid-generated turbulence, the LDV correlation measurements were compared to the longitudinal and transverse correlations of the von Kármán model (Hinze, 1975):

$$
f(r)=D_{1 / 3}\left(\kappa_{e} r\right)
$$

and

$$
g(r)=\frac{4}{3} D_{1 / 3}\left(\kappa_{e} r\right)-\frac{1}{3} D_{4 / 3}\left(\kappa_{e} r\right)
$$

where $\kappa_{e} \approx 0.75 / L_{11}$ is the wavenumber associated with the energy-containing eddies. The functions $D_{1 / 3}$ and $D_{4 / 3}$ are part of a family of functions $D_{\nu}$ that decay from one to zero, defined by

$$
D_{\nu}(x)=\frac{2^{1-\nu}}{\Gamma(\nu)} x^{\nu} K_{\nu}(x)
$$

where $\Gamma(x)$ is the Euler Gamma function and $K_{\nu}(x)$ is a modified Bessel function. Figures 3 and 4 show example comparisons of the longitudinal and transverse correlation functions. 


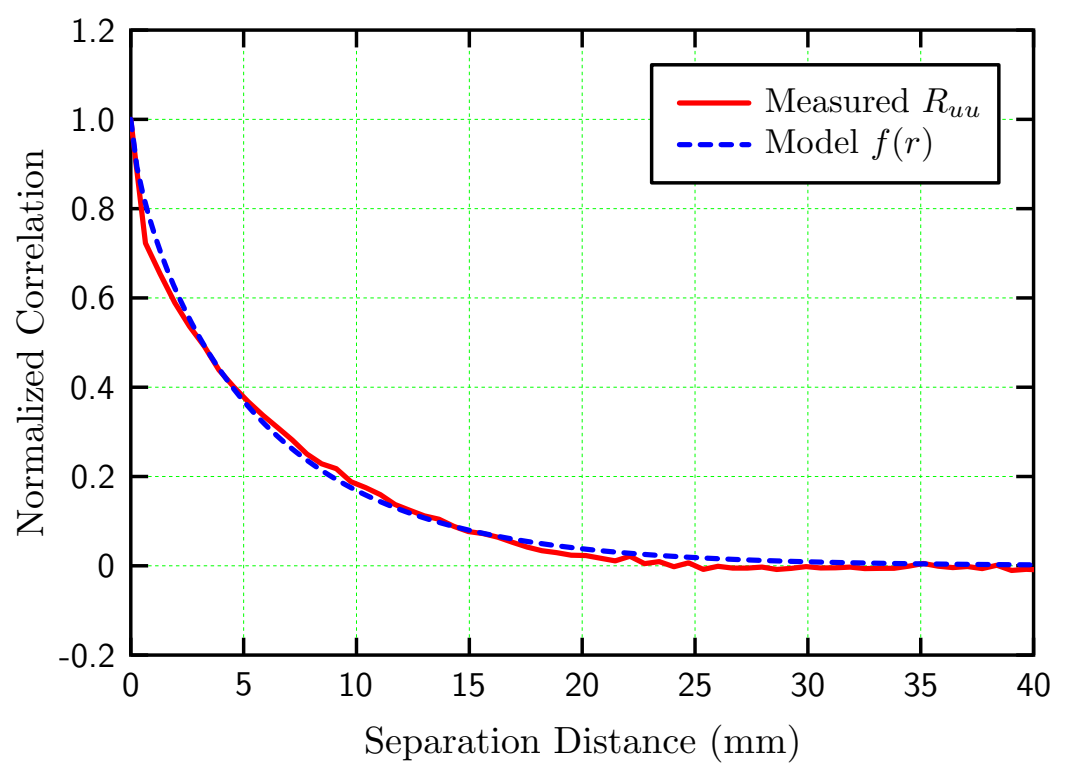

Figure 3: Measured longitudinal correlation function based on the streamwise velocity at $50 \%$ span and $3.05 \mathrm{~m} / \mathrm{s}$ (nominal), compared to model with $L_{11}=5.4 \mathrm{~mm}$.

The models $f(r)$ and $g(r)$ provide a good fit to the measured longitudinal and transverse correlations. The integral length scale for each case was then determined from a least squares fit of the model to the measured data. The best-fit integral length scales for the longitudinal and transverse correlations are different, which again indicates that the turbulence is not perfectly isotropic. To get a better idea of the variability of the length scale estimates, the least squares fit was performed at three measurement locations $(25 \%$, $50 \%$, and $75 \%$ span) and two speeds (nominally $3.05 \mathrm{~m} / \mathrm{s}$ and $7.62 \mathrm{~m} / \mathrm{s}$ ). The results are listed in Table 2 . The longitudinal correlation estimate of $L_{11}$ varied by about $\pm 15 \%$, with an average value of $4.7 \mathrm{~mm}$. For the transverse correlation, the estimate of $L_{11}$ (listed in the table as $2 L_{22}$ to distinguish it) was $3.7 \mathrm{~mm}$, with about $\pm 5 \%$ variation. Thus, on average there is about $25 \%$ difference between the longitudinal and transverse correlation estimates of the integral length scale, which gives a degree of anisotropy similar to the amount observed in the rms values.

To check the integral length scale measurements, the results can be compared to an estimate inferred from the empirical formula of Mohamed and LaRue (1990), given in Eq. (9) above. To obtain the integral length scale from this model, first note that if the turbulence is assumed to be isotropic, the turbulent kinetic energy (per unit mass) becomes $K=(3 / 2) v_{\mathrm{rms}}^{2}$. Then, the turbulence dissipation rate $\varepsilon$ can be determined from the kinetic 


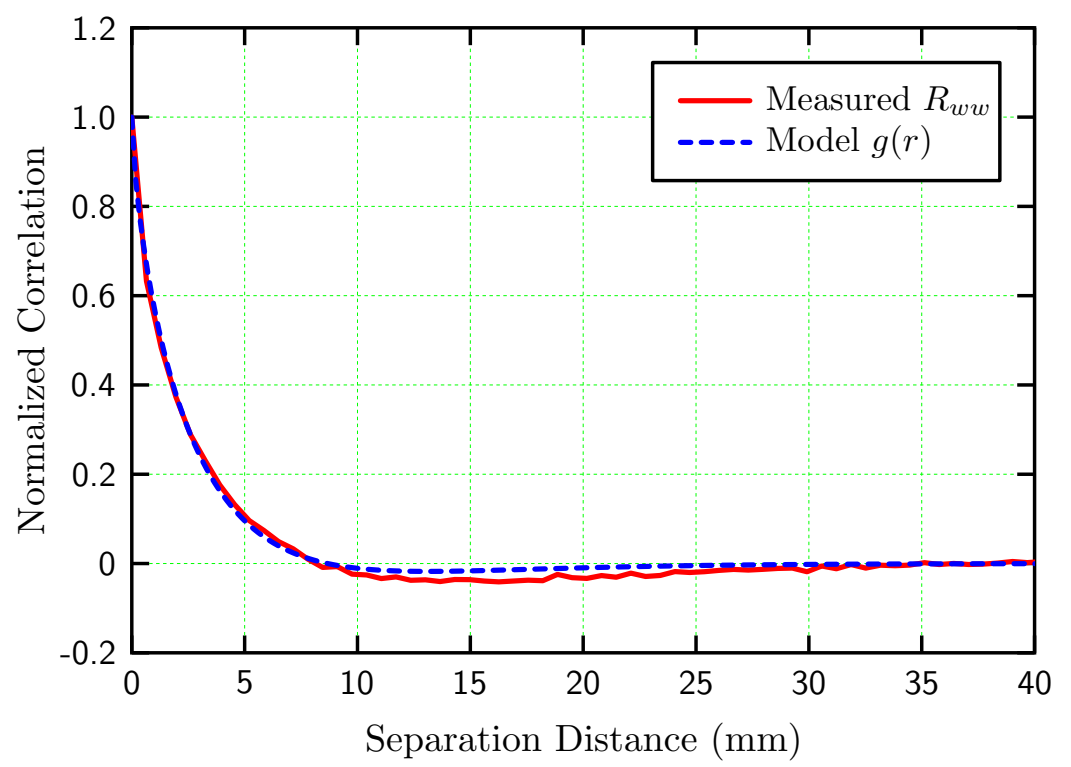

Figure 4: Measured transverse correlation function based on the spanwise velocity at $50 \%$ span and $3.05 \mathrm{~m} / \mathrm{s}$ (nominal), compared to model with $L_{11}=3.5 \mathrm{~mm}$.

Table 2: Integral length scales determined from LDV correlation measurements.

\begin{tabular}{cccc}
\hline Nominal Speed $(\mathrm{m} / \mathrm{s})$ & $z / b$ & $L_{11}(\mathrm{~mm})$ & $2 L_{22}(\mathrm{~mm})$ \\
\hline 3.05 & 0.25 & 4.7 & 3.5 \\
3.05 & 0.50 & 5.4 & 3.5 \\
3.05 & 0.75 & 4.2 & 3.8 \\
7.62 & 0.25 & 4.8 & 3.7 \\
7.62 & 0.50 & 4.2 & 3.7 \\
7.62 & 0.75 & 4.6 & 3.9 \\
\hline Average & & 4.7 & 3.7 \\
\hline
\end{tabular}


energy transport equation (Libby, 1996), which for decaying grid turbulence reduces to

$$
\varepsilon=-U_{\infty} \frac{d K}{d x}
$$

As shown by Lysak and Brungart (2003), the integral length scale can be found using

$$
L_{11} \approx 0.39 \frac{K^{3 / 2}}{\varepsilon}
$$

Substituting expressions for $K$ and $\varepsilon$ gives

$$
L_{11} \approx 0.37 M \sqrt{A}\left(\frac{x}{M}\right)^{0.35}
$$

At a distance $x=305 \mathrm{~mm}$ downstream of a grid with mesh size $M=25.4 \mathrm{~mm}$, the empirical constant range $0.035 \leq A \leq 0.055$ gives an estimated integral length scale between $4.2 \mathrm{~mm}$ and $5.3 \mathrm{~mm}$. This brackets the measured value of $L_{11}$ from the streamwise velocity, but the measured value of $2 L_{22}$ from the spanwise velocity is smaller than expected.

Based on these results and consideration of the grid geometry, it is reasonable to conclude that the vertical support bars generate larger and stronger turbulent eddies than the mesh rods, and that these eddies tend to have their axis of rotation aligned with the span. Due to their orientation, these eddies do not contribute much to the spanwise velocity fluctuations, so the rms value and integral length scale are smaller for the spanwise velocity component than for the streamwise component. However, this also suggests that the fluctuations normal to the airfoil chord, which are influenced by the support bar eddies, share the statistical properties of the measured streamwise velocities rather than the spanwise

velocities. For this reason, the streamwise measurements $v_{\mathrm{rms}} / U_{\infty}=0.047$ and $L_{11}=4.7$ $\mathrm{mm}$ will be used in the unsteady force spectrum predictions below.

\subsection{Velocity Spectra}

As a final check on the inflow turbulence, the measured longitudinal and transverse velocity spectra are compared to the corresponding von Kármán models in Figs. 5 and 6 . The longitudinal and transverse spectrum models (Hinze, 1975) are:

$$
G_{u u}(f)=4 \frac{v_{\mathrm{rms}}^{2} L_{11}}{U_{\infty}}\left[1+\left(\frac{f}{f_{e}}\right)^{2}\right]^{-5 / 6}
$$

and

$$
G_{v v}(f)=2 \frac{v_{\mathrm{rms}}^{2} L_{11}}{U_{\infty}}\left[\frac{f_{e}^{2}+\frac{8}{3} f^{2}}{f_{e}^{2}+f^{2}}\right]\left[1+\left(\frac{f}{f_{e}}\right)^{2}\right]^{-5 / 6}
$$

where $f_{e} \approx 0.12\left(U_{\infty} / L_{11}\right)$ is the frequency associated with the passing rate of energycontaining eddies. The plots show the upper and lower bounds of the six measured spectra 


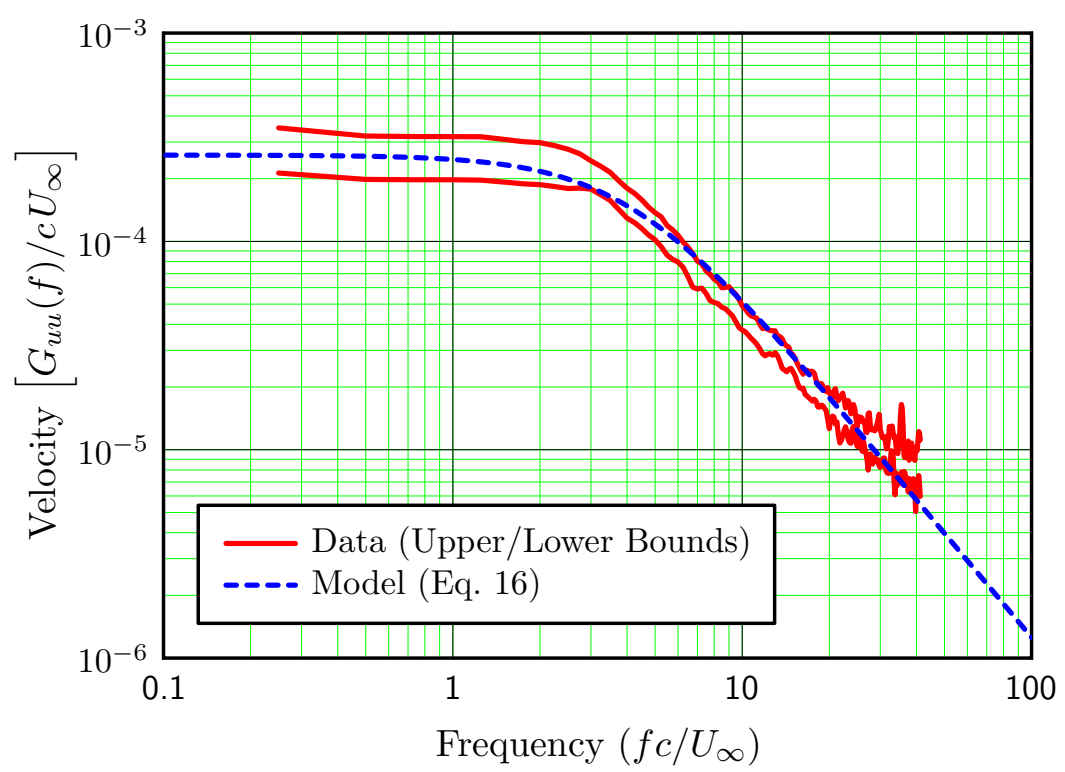

Figure 5: Measured longitudinal velocity spectrum based on the streamwise velocity, compared to model with $v_{\mathrm{rms}} / U_{\infty}=0.047$ and $L_{11}=4.7 \mathrm{~mm}$. The upper and lower bounds from six data sets are shown.

from the three locations and two speeds listed in Table 2 . In general, there is about $2 \mathrm{~dB}$ spread between the upper and lower bounds of the data. It is important to note that the uncertainty of the turbulence spectrum propagates into the uncertainty of the unsteady force spectra discussed below.

A good fit to the measured data was obtained with the longitudinal spectrum model, as shown in Fig. 5. This agreement is to be expected based on the good fit of the model to the longitudinal correlation function discussed above. For the transverse velocity spectrum, shown in Fig. 6, the comparison is based on the spanwise velocity component, so there is more discrepancy between the data and model, as expected from the lower rms values and integral length scales. The slight anisotropy of the measured turbulence therefore has a potentially significant effect on the uncertainty of the unsteady force spectrum predictions in the range $f c / U_{\infty}<3$. For the most part, this is below the frequency range where the thickness effect is important. It is also interesting to note that reasonably good agreement was obtained in the transverse spectrum for frequencies above the peak using the model with the measured streamwise velocity parameters. This region corresponds to the inertial subrange of the turbulence, where the relevant length scales of the turbulence are smaller and tend to be more isotropic. 


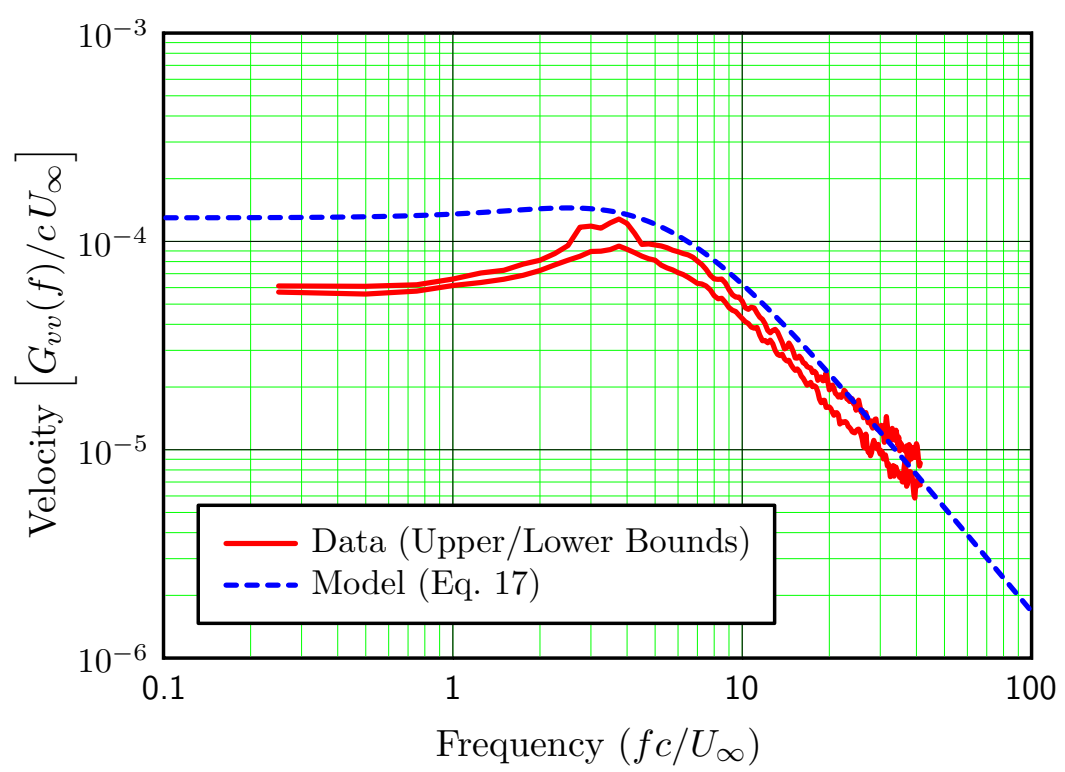

Figure 6: Measured transverse velocity spectrum based on the spanwise velocity, compared to model with $v_{\text {rms }} / U_{\infty}=0.047$ and $L_{11}=4.7 \mathrm{~mm}$. The upper and lower bounds from six data sets are shown.

This type of anisotropic effect has been examined in turbulent boundary layers by Kamruzzaman et al. (2012). If the quantity $u_{\mathrm{rms}}^{3} / L_{11}$, which is proportional to the turbulent dissipation rate, is independent of the direction of the velocity component, then the $f^{-5 / 3}$ range of the spectrum will remain isotropic, even though the low frequency range varies with direction. Another type of anisotropy, in which the length scale is stretched in a particular direction, has been modeled by Lynch et al. (2005). This would be appropriate for turbulence passing through a contraction, and it affects the entire frequency range by shifting the peak of the spectrum. In this experiment, the inertial subrange is nearly isotropic, so the anisotropic effects are limited to the low frequency range. It is also worth noting that a large part of the turbulent energy lies in the region $f c / U_{\infty}>1$, which is the frequency range of interest for the unsteady force measurements. This helps to improve the signal-to-noise ratio of these measurements.

It should also be noted that at high enough frequencies, viscous dissipation modifies the velocity spectra, resulting in a much faster dropoff than the $f^{-5 / 3}$ slope observed in the LDV results and predicted by the von Kármán model. In this experiment, the viscous range occurs well above the frequency range of interest. However, in other applications, particularly at lower Reynolds numbers, viscous attenuation can have an important influence on 
the high frequency spectrum. Roger and Moreau (2007) present spectra that extend into the viscous range and discuss modifications to the velocity spectra models that account for the faster dropoff.

\section{$5 \quad$ Unsteady Force Measurements}

Since the main objective of the water tunnel experiment was to measure the unsteady force spectrum for airfoils of different thickness-to-chord ratios, it is important that the measurements be free of system resonances which could influence the shape of the force spectrum. Also, because the water tunnel facility generates a significant amount of vibration and noise itself, the background noise needed to be considered. The noise floor was measured by testing the airfoils without the turbulence grid installed. Before obtaining the final results with the turbulence grid, a data processing procedure was developed to remove extraneous noise from the force signals and to extract the frequency range where valid results were measured. As an additional check on the accuracy of the measurements, the unsteady force spectra were obtained over a wide range of speeds and collapsed into a single non-dimensional curve.

\subsection{System Resonances and Calibration}

In the experiment, the system resonances were measured as part of the impact tests that were used to calibrate the unsteady force measurements. An impulse force hammer (PCB Piezotronics model 086M77 with a plastic tip) with a built-in force gage was used for the tests. The measurements were performed in air with the water tunnel drained, in order to have access to the installed airfoil. The impact tests were performed by tapping the airfoil with the hammer at several positions, and forming the transfer function between the input force and the output force. The output force was obtained by summing the signals from the four force gages in the airfoil bases. The results are shown in Fig. 7, which represents an average of five hit points (center, top, bottom, left, right). A nearly flat frequency response was obtained up to about $300 \mathrm{~Hz}$, with the first resonance occurring around $1000 \mathrm{~Hz}$.

In the flat frequency response range, the ratio of the output force to the input force is approximately equal to one, which confirms that nearly all the force is being transmitted through the force gages. The slight departures from the ideal ratio of one are partly a result of the small differences in the actual sensitivity of the force gages compared to the nominal value. In addition, there were some differences in the results obtained for the different airfoils, which could be due to slight changes in the force gage contact points, since each airfoil fit between the bases slightly differently. The impact test results in the flat frequency 


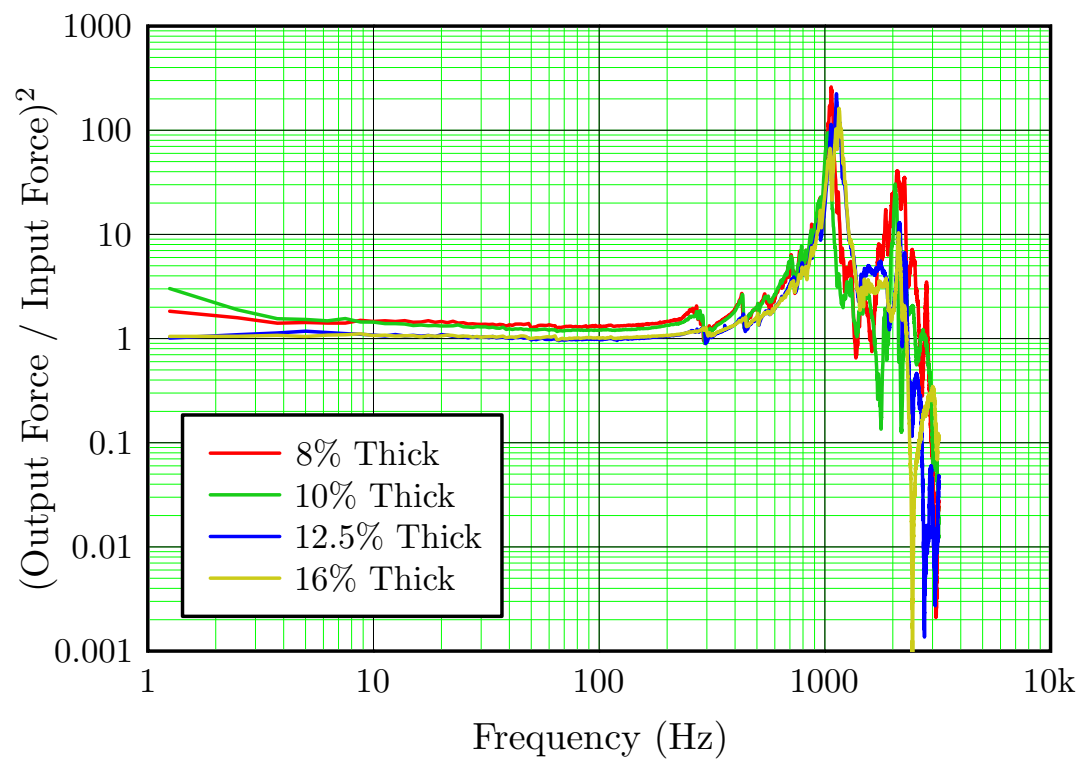

Figure 7: Calibration of the summed force gage response with different airfoils installed.

range were used to provide a small calibration correction factor (up to $1.8 \mathrm{~dB}$ ) to the forces measured in the subsequent flow tests.

One of the reasons for using four force sensors was that it made the measurement of the net force more robust. While the output of the individual force sensors depended on the hit point location, the sum was essentially the same for all hit points, at least in the flat frequency response range. For example, the force gages at the top of the airfoil responded more strongly when the airfoil was struck near the top than when it was struck near the bottom, but the sum of all four sensors was unchanged. However, at higher frequencies, where the results are affected by the resonances of the system, even the summed output force was found to depend on the hit point location. While an average of several hit points helps to reduce the sensitivity, application of a calibration factor in this situation would need to consider the expected loading distribution on the airfoil. Also, it should be noted that in order to use the high frequency region, the calibration would need to be performed in water (with a remote-controlled impact hammer) in order to account for the frequency shift due to the added mass effect. Therefore, in this experiment, only the flat frequency response region was retained in the force measurements. 


\subsection{Data Processing and Background Noise Removal}

The unsteady force measurements were made by digitally recording the individual sensor signals for 100 seconds at a sampling rate of $20480 \mathrm{~Hz}$. A sixteen channel National Instruments data acquisition system running a custom-designed LabView application was used to acquire the data, and the post-processing was performed using Matlab. To calculate the force spectra, the force sensor signals were first summed in the time domain, and then the autospectrum was computed using the method detailed in Bendat and Piersol (1986). The spectra were computed using a blocksize of 16384, giving a frequency resolution of $1.25 \mathrm{~Hz}$, with a Hanning window and $50 \%$ overlap.

Before obtaining the final results, some additional data processing was applied to reduce contamination from the background noise of the facility. This noise arises from vibration produced by the motor used to drive the water tunnel and from other machinery in the building, as well as from flow noise from the tunnel walls and the turbulence-generating grid. Fortunately, a substantial portion of the frequency range of interest had minimal noise contamination and it was relatively easy to identify where noise was present.

To eliminate the machinery noise, accelerometers were mounted on the outside of the test section to monitor the vibration. A total of four accelerometers were placed on the top and bottom window inserts, the side of the test section, and the test section flange. An example of the accelerometer spectra is shown in Fig. 8. It can be noticed that the accelerometer signals are dominated by tonal noise associated with the facility machinery. It was assumed that the portion of the force signal that was coherent with the accelerometers was related to facility noise. A multiple coherence noise removal technique, which is related to the conditioned spectrum described by Bendat and Piersol (1986), was used to remove the portion of the signal detected by the accelerometers from the force signal. A similar method was successfully used to remove vibration contamination from wall pressure spectra by Bonness and Jenkins (2012).

Figure 9 shows the effectiveness of this approach. The original measured force has a significant amount of tonal noise contamination at higher frequencies due to the tunnel drive system, as well as some contamination at lower frequencies. Using a single accelerometer, a simple coherent noise removal approach can be implemented as

$$
\hat{G}_{F F}=\left(1-\gamma_{F a}^{2}\right) G_{F F}
$$

where $\hat{G}_{F F}$ is the corrected force spectrum, $\gamma_{F a}^{2}$ is the coherence between the force and accelerometer signals, and $G_{F F}$ is the measured force spectrum. This is effective in reducing some of the noise, but better results are obtained by using additional accelerometers since different locations may detect different parts of the background vibration. 


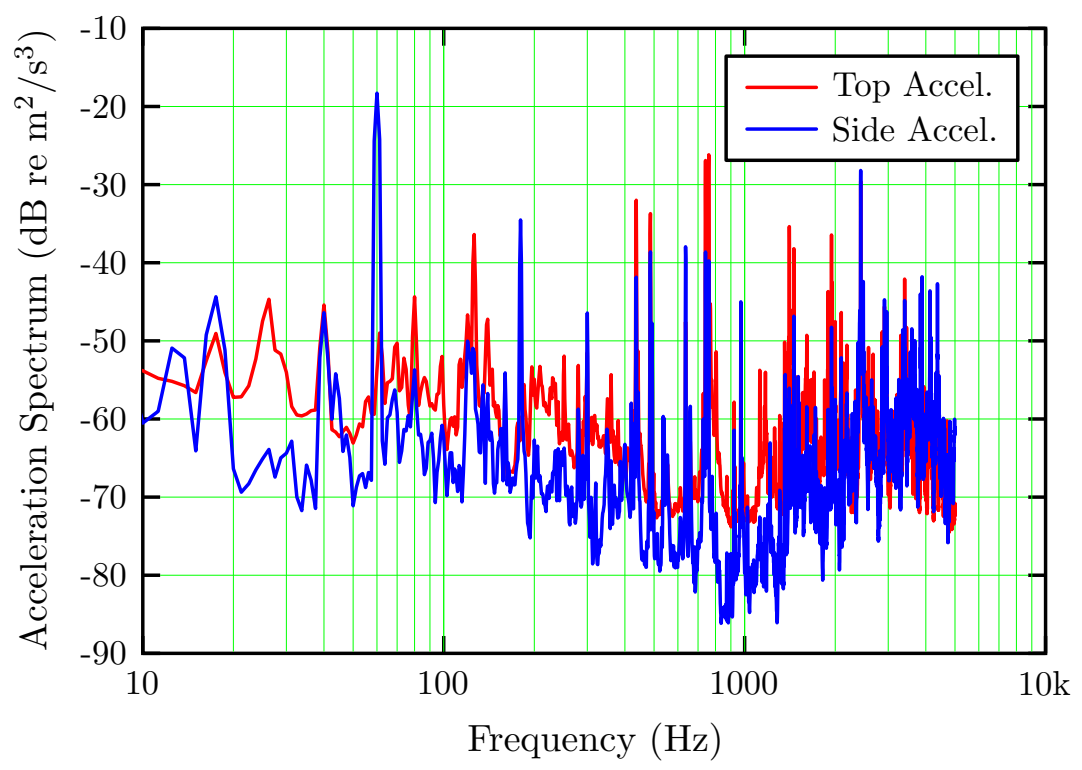

Figure 8: Example accelerometer spectra. Data from the $12.5 \%$ thick airfoil test at 12.5 $\mathrm{ft} / \mathrm{s}$ (nominal) are shown.

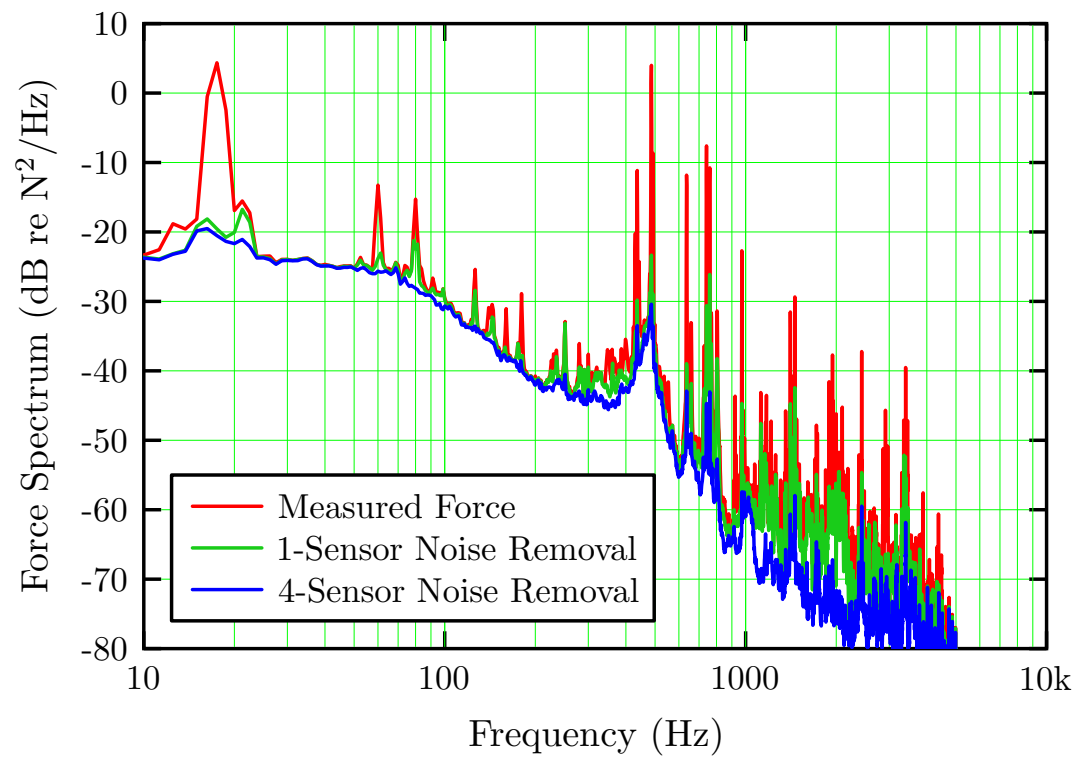

Figure 9: Example results of the coherent noise removal technique. Data from the 12.5\% thick airfoil at $12.5 \mathrm{ft} / \mathrm{s}$ (nominal) are shown. 
The multiple coherence noise removal technique uses the full cross-spectrum matrix between the all the signals, and iteratively removes the portion of the force that is coherent with the accelerometer signals. To implement this technique, start with the matrix $G_{i j}(f)$, which represents the cross-spectrum between signals $x_{i}(t)$ and $x_{j}(t)$. The rows and columns are arranged such that range $i=1 \ldots n-1$ corresponds to the noise reference signals (in this case, the accelerometers) and $i=n$ corresponds to the output signal (the summed force). The conditioned cross-spectrum matrix is obtained by evaluating the following equation in a loop over $k=1 \ldots n-1$ :

$$
G_{i j} \leftarrow G_{i j}-\frac{G_{k j}}{G_{k k}} G_{i k}
$$

Each iteration uses the partial coherence function to remove the portion of the other signals that is coherent with signal $x_{k}(t)$. Upon completion, the matrix entry $G_{n n}(f)$ contains the output spectrum with the coherent noise removed. It can be seen in Fig. 9 that this approach is very effective in removing the tonal noise contamination from the measured force spectrum.

Once the force signal is cleaned up, it is easier to identify where the flow noise of the facility limits the measurement of the turbulence induced forces. To determine whether the measured force was produced by the grid turbulence, measurements were made with and without the turbulence-generating grid installed. Fig. 10 shows a typical result. Below about $100 \mathrm{~Hz}$, there is at least $10 \mathrm{~dB}$ signal-to-noise. However, above $300 \mathrm{~Hz}$ the measured force appears to be controlled by facility flow noise, as the spectrum is nearly the same with and without the grid. The first resonance of the airfoil, which was near $1000 \mathrm{~Hz}$ in air, appears to shift down to around $450 \mathrm{~Hz}$ due to the added mass of the water. The vibration of the grid itself appears to be noticeable above $1000 \mathrm{~Hz}$.

Based on the signal-to-noise analysis and the flat region of the force calibration, the usable frequency range was determined to be 10-250 Hz. Unsteady force measurements were made at nominal speeds of $10,12.5,16,20,25$, and $31.5 \mathrm{ft} / \mathrm{s}(3.05,3.8,4.9,6.1,7.6$, $9.6 \mathrm{~m} / \mathrm{s}$ ), corresponding to a Reynolds number based on chord of $0.5 \times 10^{6}$ to $1.6 \times 10^{6}$. The speed distribution was selected to follow an ANSI preferred number series (Jonson, 2007). Since the preferred number series is proportionally based, a uniformly spaced stack-up of the force spectrum is obtained when plotted on log-log plot. The same methodology was applied in choosing the thicknesses of the airfoils. The spectral density between 10-250 $\mathrm{Hz}$ was extracted from these measurements and divided by the calibration factor shown in Fig. 7 to get the force acting on the airfoil. The results were then normalized to collapse the data at different speeds to a single curve. The resulting non-dimensional spectrum $G_{F F}(f) /\left(\rho^{2} U_{\infty}^{3} c^{5}\right)$ depends only on the normalized frequency $\left(f c / U_{\infty}\right)$, the turbulence intensity $\left(v_{\mathrm{rms}} / U_{\infty}\right)$, the ratio of the integral length scale to the airfoil chord $\left(L_{11} / c\right)$, the 


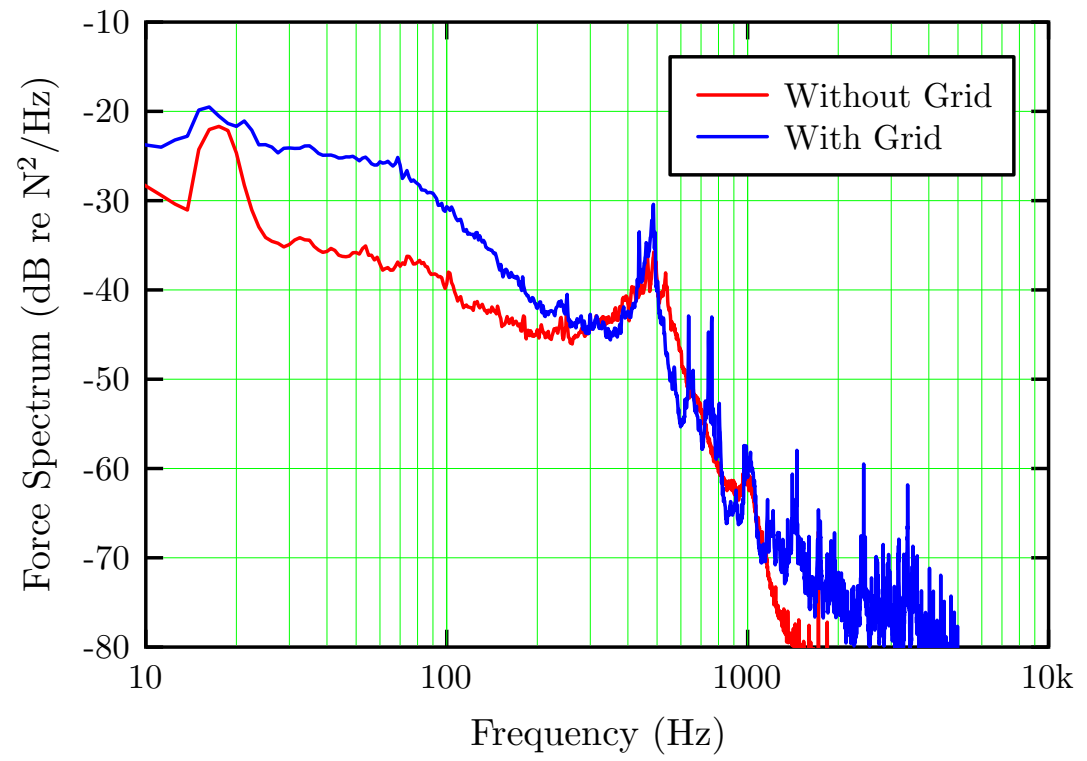

Figure 10: Example signal-to-noise results. Data from the $12.5 \%$ thick airfoil at $12.5 \mathrm{ft} / \mathrm{s}$ (nominal) are shown.

aspect ratio of the airfoil $(b / c)$, and the thickness-to-chord ratio $(\tau / c)$. As shown in Fig. 11, the data collected at different speeds collapsed well to a single non-dimensional curve.

For the thicker airfoil cases, the background noise level (as determined from the data without the turbulence grid) is within $6 \mathrm{~dB}$ of the measured spectrum at the high-frequency end of the spectrum. In such situations, it is appropriate to apply a background noise correction (Pierce, 1989) by subtracting the background level from the measured spectrum. Because the background data were obtained at different speeds than the measured data (due to the removal of the turbulence-generating grid), it is essential that the background correction be applied to the normalized spectrum. This was implemented by fitting a curve to the normalized background spectrum, and subtracting this curve from the measured spectrum. Figures 12 and 13 show the background corrections for the $12.5 \%$ and $16 \%$ thick airfoil data. The curve fits are based on the function

$$
G(f)=a\left(f c / U_{\infty}\right)^{b}
$$

The parameters $a$ and $b$ were found from a logarithmic least squares fit of the data in the frequency range $4 \leq f c / U_{\infty} \leq 10$. This gave the values $a=2.1 \times 10^{-7}$ and $b=-1.7$ for the $12.5 \%$ thick airfoil, and $a=1.4 \times 10^{-6}$ and $b=-2.4$ for the $16 \%$ thick airfoil. The background noise correction applied to the measured spectrum was quite small for the 


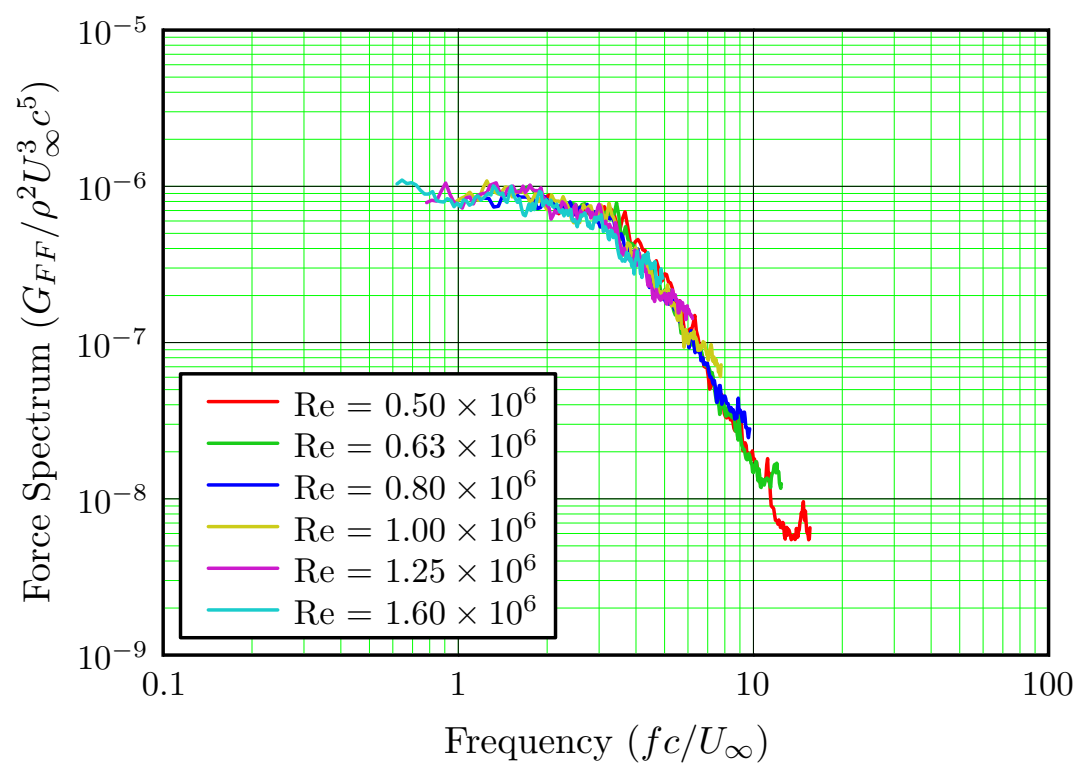

Figure 11: Collapse of the measured force spectra to a single non-dimensional curve. Data from the $12.5 \%$ thick airfoil is shown for a series of Reynolds numbers.

$12.5 \%$ airfoil, except in the frequency range $f c / U_{\infty}>10$ where it lowered the results by several $\mathrm{dB}$. For the $16 \%$ thick airfoil, the correction is noticeable above $f c / U_{\infty}>7$, and the measurement was background limited (meaning that the corrected results would be lower than the background level) for $f c / U_{\infty}>10$. The background limited data were discarded, so the corrected spectrum only extends up to $f c / U_{\infty}=10$ for the $16 \%$ thick airfoil. For the $8 \%$ and $10 \%$ thick airfoils, the background spectra are more than $8 \mathrm{~dB}$ below the measured spectra, so the background corrections are negligible.

\section{Results}

The measured results for the four airfoils of different thickness-to-chord ratios are shown in Figs. 14-17. For each airfoil tested, a normalized force spectrum was obtained by collapsing the data from different speeds. Also shown on the plots is the spectrum predicted by the analytical model given in Eq. (1), with and without the modified gust response based on the effect of thickness given by Eq. (7). To make the calculations, the turbulence intensity was assumed to be $4.7 \%$ and the integral length was assumed to be $4.7 \mathrm{~mm}$, based on the statistics obtained from the streamwise velocity component of the LDV measurements. As noted above, the accuracy of the overall model depends in large part on the accuracy of 


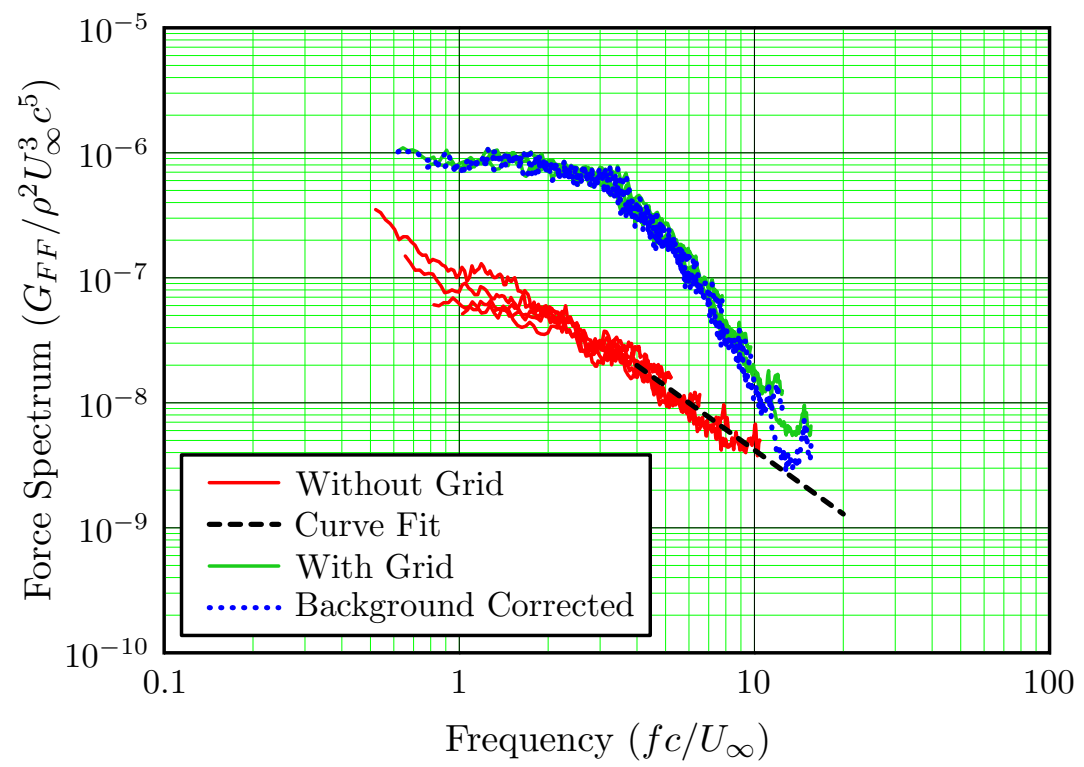

Figure 12: Background noise correction applied to the $12.5 \%$ thick airfoil.

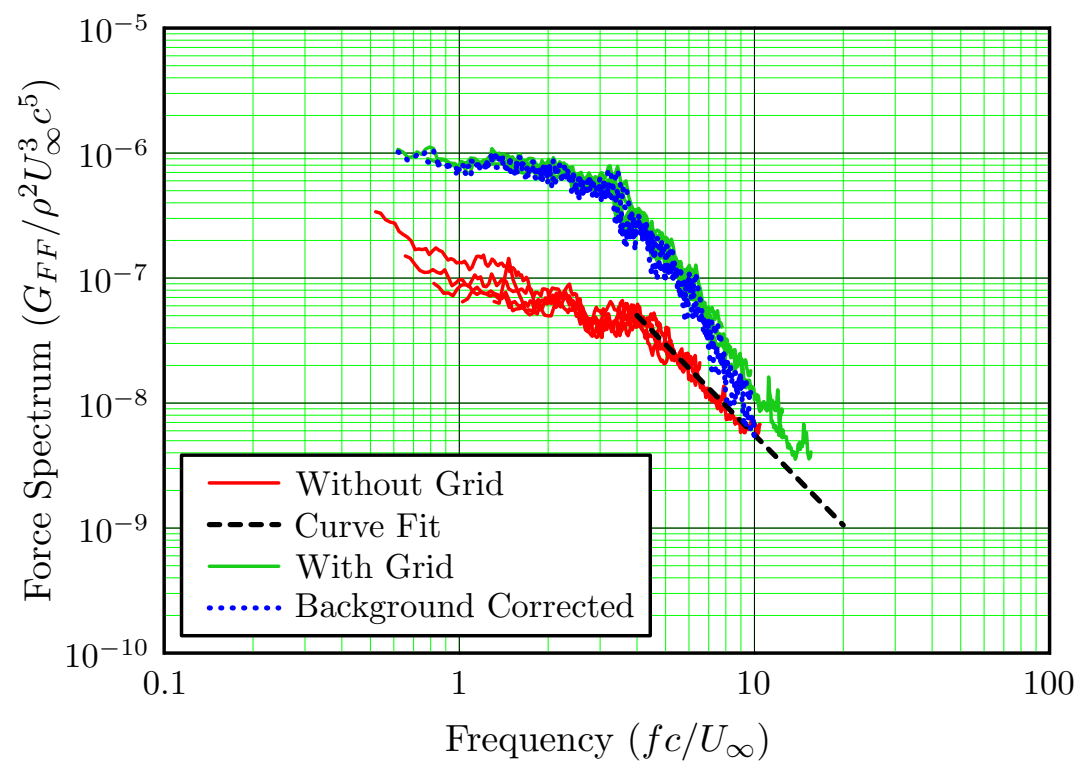

Figure 13: Background noise correction applied to the $16 \%$ thick airfoil. 


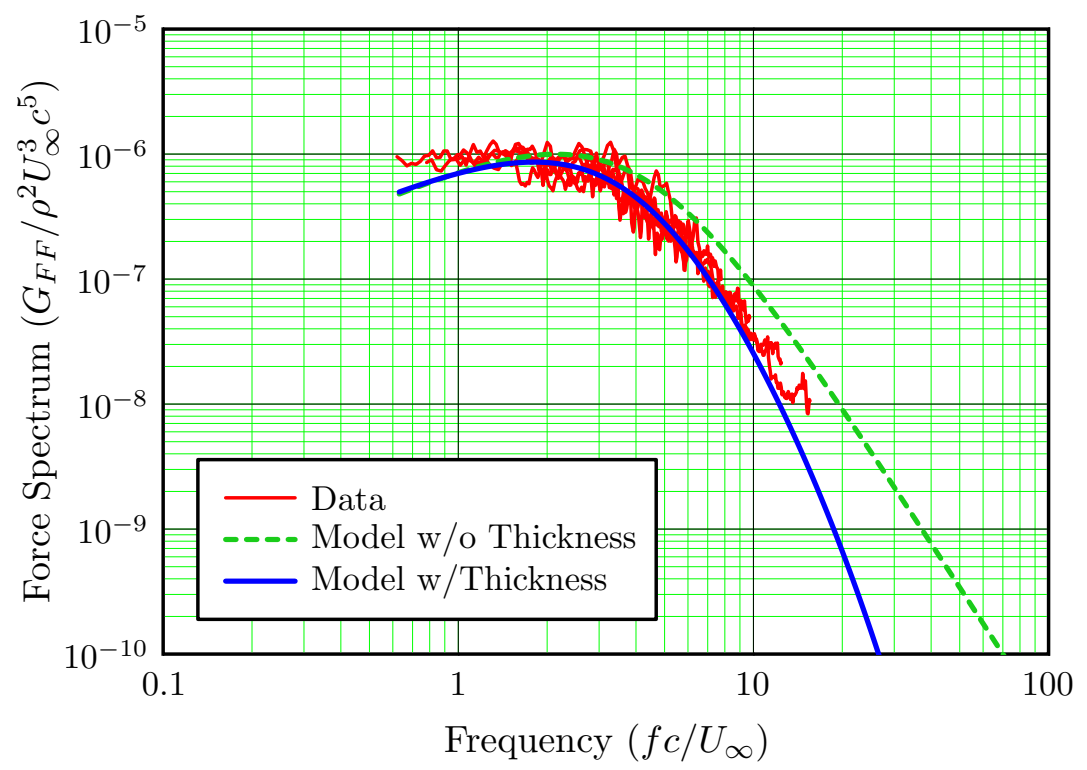

Figure 14: Force spectrum results for the $8 \%$ thick airfoil.

the turbulence parameters used in the model. However, relative comparisons between the different airfoils are not affected, since the same turbulence spectrum was used for each airfoil.

Overall, there is good agreement between the experimental results and the analytical model calculations that include the thickness correction. Without the thickness correction, significant overprediction of the measured force occurs in the high frequency range. Thus, the experimental measurements clearly demonstrate the importance of including the thickness effect in modeling the high frequency gust response, consistent with previous wind tunnel radiated noise results in the literature. Furthermore, comparing the results as the thickness increases from $8 \%$ to $16 \%$, the rate of the high frequency roll-off increases with thickness. This effect is captured accurately by the predictions, which validates use of Eq. (8) for this family of airfoils.

The absolute level of the force spectrum was found to be predicted well using the measured turbulence parameters from the streamwise velocity component. There is some uncertainty if this accurately represents the gust spectrum due to the slight anisotropy of the turbulence. However, the turbulence was found to be nearly isotropic for $f c / U_{\infty}>3$, and there is no apparent change in the accuracy of the model prediction between the lower frequencies where anisotropic effects appear in the velocity spectra and higher frequencies that are isotropic. It is possible that the streamwise turbulence parameters do not exactly 


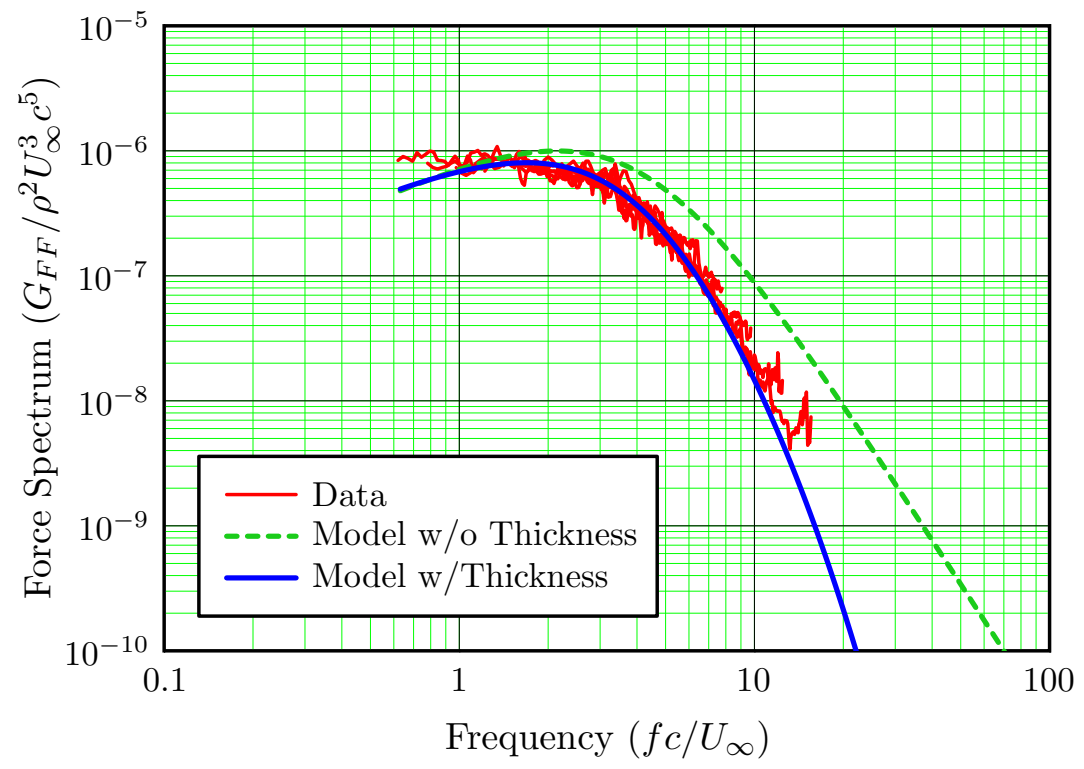

Figure 15: Force spectrum results for the $10 \%$ thick airfoil.

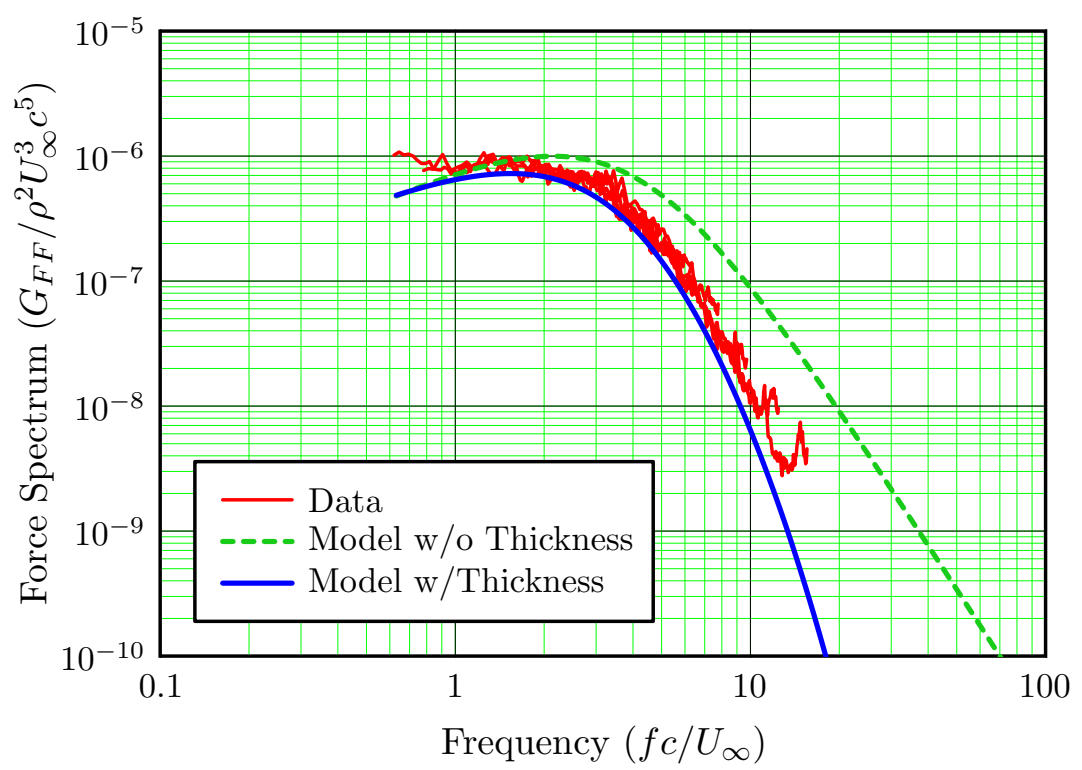

Figure 16: Force spectrum results for the $12.5 \%$ thick airfoil. 


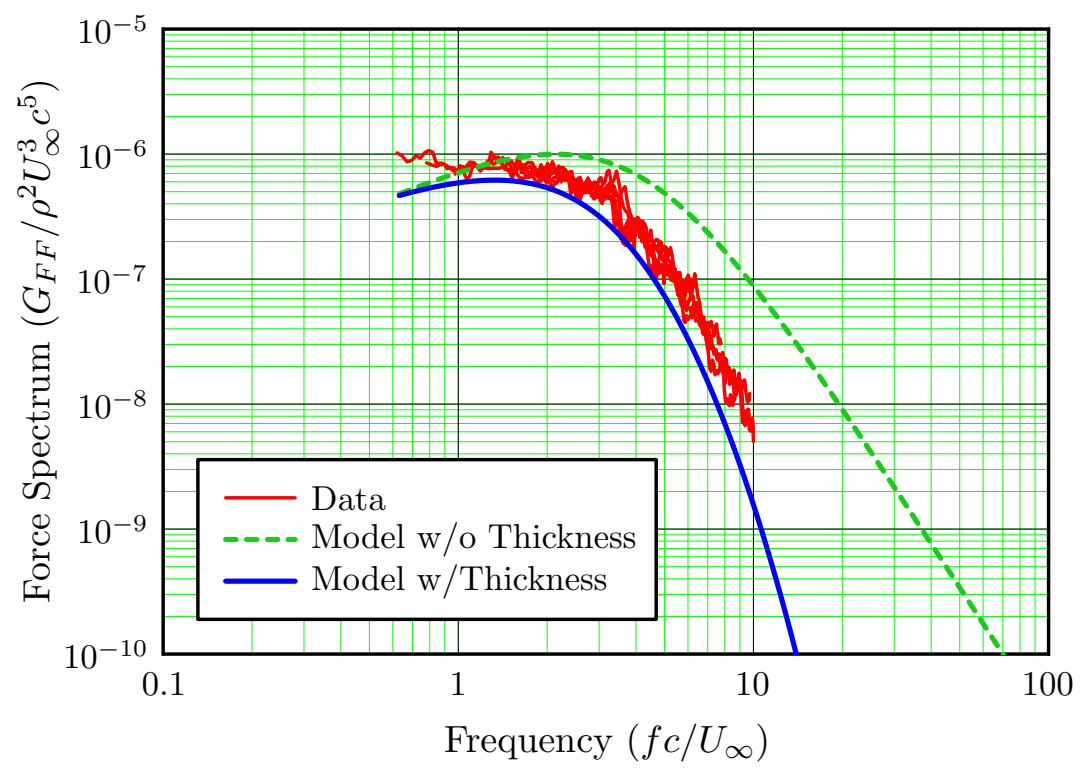

Figure 17: Force spectrum results for the $16 \%$ thick airfoil.

represent the upstream gust, but that the distortion of the gust as it approaches the airfoil changes the anisotropy of the turbulence. This idea could perhaps be examined with a model that uses thickness to change the inflow spectrum rather than the airfoil gust response, as in Moreau et al. (2005). A complete understanding of the effects of the turbulence anisotropy on the unsteady forces will require further investigation.

Also, the agreement between the predictions and the model is not quite as good for the thickest two foils. This could be just a limitation of the force calibration, as Fig. 7 shows variations of about $2 \mathrm{~dB}$ when the different airfoils were installed. However, there does appear to be a systematic trend, so it might suggest that the model slightly overpredicts the thickness attenuation as the thickness increases.

\section{Conclusion}

A water tunnel experiment that measured the unsteady lift on a series of airfoils subjected to grid-generated turbulence was performed to determine the effect of thickness on high frequency gust response. The test setup made use of four piezoelectric force gages at the connection points between the airfoil bases and the test section frame. Calibration of the system using an impulse force hammer showed that the sum of the force gage signals returned the total input force, with a flat frequency response in the range of inter- 
est. Contamination from facility vibration was reduced using a multiple coherence noise removal technique using accelerometers mounted on the outside of the test section. Measurements were made over a wide range of speeds and found to collapse well into a single non-dimensional force spectrum. The inflow turbulence was measured using Laser Doppler Velocimetry, and was found to be reasonably well approximated by an isotropic, homogeneous turbulence spectra model. The measured force spectra were found to be in good agreement with theory when an appropriate thickness correction factor was applied. In particular, the results show the importance of accounting for the airfoil thickness to prevent significant overprediction of the forces in the high frequency range.

\section{Acknowledgements}

This work was supported by the Office of Naval Research under contract N00014-05G106/0090, project monitor Debbie Nalchajian, Code 331, and by the Exploratory and Foundational Research Program of the Pennsylvania State University Applied Research Laboratory. The authors would also like to thank Dr. Michael Krane for his help in conducting the Laser Doppler Velocimetry measurements.

\section{References}

[1] Abbott, I.H., von Doenhoff, A.E., 1959. Theory of Wing Sections. Dover Publications.

[2] Amiet, R.K., 1975. Acoustic radiation from an airfoil in a turbulent stream. Journal of Sound and Vibration 41, 407-420.

[3] Amiet, R.K., 1976. High frequency thin-airfoil theory for subsonic flow. AIAA Journal 14, 1076-1082.

[4] Bendat, J.S., Piersol, A.G., 1986. Random Data: Analysis and Measurement Procedures, 2nd ed. Wiley.

[5] Benedict, L.H., Nobach, H., Tropea, C., 2000. Estimation of turbulent velocity spectra from laser doppler data. Measurement Science and Technology 11, 1089-1104.

[6] Bonness, W.K., Jenkins, D.M., 2012. Coherent noise removal from dynamic wall pressure and vibration signals. Proceedings of InterNoise 2012, IN12-1534.

[7] Devenport, W.J., Staubs, J.K., Glegg, S.A.L., 2010. Sound radiation from real airfoils in turbulence. Journal of Sound and Vibration 329, 3470-3483. 
[8] Gershfeld, J., 2004. Leading edge noise from thick foils in turbulent flows. Journal of the Acoustical Society of America 116, 1416-1426.

[9] Hinze, J.O., 1975. Turbulence, 2nd ed. McGraw-Hill.

[10] Jonson, M.L., 2007. Preferred vehicle scaling. Proceedings of the ASME International Mechanical Engineering Congress, IMECE2007-41135.

[11] Kamruzzaman, M., Lutz, T., Herrig, A., Kramer, E. 2012. Semi-empirical modeling of turbulent anisotropy for airfoil self-noise predictions. AIAA Journal 50, 46-60.

[12] Lehman, A.F., 1959. The Garfield Thomas Water Tunnel. Report NORD 16597-56, The Pennsylvania State University, University Park, PA.

[13] Libby, P.A., 1996. Introduction to Turbulence. Taylor \& Francis.

[14] Lynch, D.A., Blake, W.K., Mueller, T.J., 2005. Turbulence correlation length-scale relationships for the prediction of aeroacoustic response. AIAA Journal 43, 1187-1197.

[15] Lysak, P.D., Brungart, T.A., 2003. Velocity spectrum model for turbulence ingestion noise from computational-fluid-dynamics calculations. AIAA Journal 41, 1827-1829.

[16] Lysak, P.D., 2011. Unsteady Lift of Thick Airfoils in Incompressible Turbulent Flow. Ph.D. Dissertation, Graduate Program in Acoustics, The Pennsylvania State University.

[17] Lysak, P.D., Capone, D.E., Jonson, M.L., 2013. Prediction of high frequency gust response with airfoil thickness effects. Journal of Fluids and Structures 39, 258-274.

[18] Mohamed, M.S., LaRue, J.C., 1990. The decay power law in grid-generated turbulence. Journal of Fluid Mechanics 219, 195-214.

[19] Moreau, S., Roger, M., Jurdic, V., 2005. Effect of angle of attack and airfoil shape on turbulence-interaction noise. Proceedings of the 11th AIAA/CEAS Aeroacoustics Conference, AIAA Paper 2005-2973.

[20] Moreau, S., Roger, M., 2007. Competing broadband noise mechanisms in low-speed axial fans. AIAA Journal 45, 48-57.

[21] Moriarty, P.J., Guidati, G., Migliore, P., 2005. Prediction of turbulent inflow and trailing-edge noise for wind turbines. Proceedings of the 11th AIAA/CEAS Aeroacoustics Conference, AIAA Paper 2005-2881. 
[22] Oerlemans, S., Migliore, P., 2004. Aeroacoustic wind tunnel tests of wind turbine airfoils. Proceedings of the 10th AIAA/CEAS Aeroacoustics Conference, AIAA Paper 2004-3042.

[23] Olsen, W., Wagner, J., 1982. Effect of thickness on airfoil surface noise. AIAA Journal $20,437-439$.

[24] Paterson, R.W., Amiet, R.K., 1977. Noise and surface pressure response of an airfoil to incident turbulence. Journal of Aircraft 14, 729-736.

[25] Petrie, H.L., 1995. Reduction of noise effects on power spectrum estimates using the discretized lag product method. Proceedings of the ASME/JSME Fluids Engineering and Laser Anemometry Conference, FED 229, 139-144.

[26] Pierce, A.D., 1989. Acoustics: An Introduction to Its Physical Principles and Applications. Acoustical Society of America.

[27] Roger, M., Moreau, S., 2010. Extensions and limitations of analytical airfoil broadband noise models. International Journal of Aeroacoustics 9, 273-305.

[28] Sears, W.R., 1941. Some aspects of non-stationary airfoil theory and its practical application. Journal of the Aeronautical Sciences 8, 104-108. 